\title{
El papel de la alta formación escolar en la inserción de las mujeres migrantes mexicanas
}

\author{
Salvatori,Sara \\ Scalabrini International Migration Institute, Roma,Italia \\ sarasalvatori@hotmail.com \\ Terrón Caro,Teresa \\ Universidad Pablo de Olavide, Sevilla,España \\ mttercar@upo.es
}

\section{Resumen}

En una época en la que la movilidad de los individuos es parte estructural del contexto en el que hombres y mujeres actúan, nos preguntamos si la posesión de un alto nivel escolar incide en la inserción de los sujetos en las sociedades receptoras. Para ser más precisos nos interrogamos sobre si la alta formación profesional puede ser un parámetro para determinar la inclusión de las y los migrantes en el mercado laboral de los países de llegada.

Para contestar a nuestras preguntas, consideramos pertinente el uso del enfoque interseccional basado en el análisis relacional del género, la posición social y el origen nacional con el objetivo de generar un punto de vista que contemple los diferentes niveles del análisis.

El contexto de estudio es el incluido entre el espacio social transnacional Monterrey (México)- Houston (Estados Unidos) donde, con el propósito de desarrollar un análisis cualitativo, se han entrevistado mujeres mexicanas migrantes con estudios universitarios.

Los datos recogidos muestran que el nivel escolar, así como la posición social no son elementos que pueden fácilmente transnacionalizarse, es decir no tienen la capacidad de cruzar fronteras, al contrario del origen nacional que termina por caracterizar las mujeres mexicanas estigmatizándolas. En efecto, se trata de un proceso que se construye en el interior de las esferas políticas y económicas, donde la inserción de las y los migrantes no depende del capital cultural poseído sino de las necesidades del sistema productivo.

\section{Abstract}

At a time when the mobility of individuals is a structural part of the context in which men and women act, we wonder whether the possession of a high school level affects the insertion of subjects in the receiving societies. To be more precise, we ask ourselves whether high professional training can be a parameter to determine the inclusion of migrants in the labor market of the countries of arrival.

To answer these questions, we consider relevant the use of the intersectional approach based on the relational analysis of gender, social position and national origin in order to generate a point of view that considers the different levels of analysis.

The study context is the one included between the transnational social space Monterrey (Mexico) - Houston (UnitedStates) where, with the purpose to develop a qualitative analysis, Mexican skilled migrant women have beeninterviewed.

The data collected show that the school level, as well as the social position, are not elements that can be easily transnationalized, that is, they do not have the capacity to cross borders, contrary to the national origin that ends up characterizing Mexican women stigmatizing them. It is a process that is built within the political and economic spheres, where the insertion of migrants does not depend on the cultural capital possessed but on the needs of the productive system.

Palabras clave: migración, género, posición social, origen nacional, México-Estados Unidos.

Keywords: migration, gender, social position, national origin, Mexico-United States. 


\section{INTRODUCCIÓN}

La presente comunicación es parte del trabajo de investigación para la tesis doctoral titulada «La migración invisibilizada de mujeres cualificadas de Monterrey (México) a Houston (Texas): una interpretación desde el enfoque interseccional ${ }^{1} »$. Se trata de un estudio cualitativo centrado en la experiencia migratoria de mujeres con un alto nivel escolar que, siguiendo rutas transnacionales, se insertan en determinados sectores laborales en el país de llegada. En particular, en este contexto pretendemos reflexionar sobre si la posesión de un alto nivel escolar incide en la inserción de los sujetos en las sociedades receptoras. Para ser más precisos, nos interrogamos sobre si la alta formación profesional puede ser un parámetro para determinar la inclusión de las y los migrantes en el mercado laboral calificado en el lugar de asentamiento.

Para contestar a nuestra pregunta, primero nos centramos en el enfoque interseccional para poder generar conocimiento a partir del análisis de género, de la posición social y del origen nacional, seguimos presentando algunos datos sobre la migración de las mujeres mexicanas con un alto nivel escolar y terminamos analizando las dinámicas que afectan su inserción laboral a la luz de la interrelación de los elementos que conforman el enfoque interseccional.

\section{ACERCAMIENTO TEÓRICO}

Con el propósito de contestar a la pregunta de investigación consideramos útil fundamentar nuestro análisis a partir del enfoque interseccional. Sin embargo, creemos oportuno también reinterpretar este enfoque a la luz de las características que distinguen los estudios migratorios. Para aclarar nuestro punto de partida, primeramente necesitamos especificar que el enfoque interseccional nace en los años 70 cuando las feministas norteamericanas utilizan el análisis de género, de clase y de etnia para poder estudiar la triple opresión padecida por las mujeres afro- americanas. Precisamente, es la interrelación de los elementos que determina el aporte epistemológico necesario para la comprensión del fenómeno analizado (Anthias y Yuval-Davis, 2010).

Ahora bien, según nuestro punto de vista el análisis desde el enfoque interseccional bien puede aplicarse al estudio de los fenómenos migratorios aunque requiere la adaptación a un objeto que presenta una amplia variedad de casos no sólo en relación a los contextos de procedencia, sino también en relación a las diferencias de género, sociales, culturales y económicas que distinguen los individuos involucrados en los movimientos migratorios.

Para ello, nuestra propuesta radica en adaptar el modelo estratificador construido a partir de la intersección del género, la clase y la etnia a contextos culturales y económicos no necesariamente afines al norteamericano para el cual este enfoque ha sido diseñado. Así que,lo que proponemos es por un lado desligar el enfoque interseccional del concepto de clase social que indica un determinado modelo de producción, y por el otro, añadir al concepto de etnia algún otro elemento capaz de enriquecer el análisis.

Siguiendo esta línea de investigación, optamos en primer lugar por el uso del concepto de posición social a medida que identifica un sistema estratificado dentro de una estructura social (Seymour- Smith, 1986) que no necesariamente se reduce a la individuada por la división en clases. En definitiva,se trata de la representación del espacio social a partir de las características y roles que ubican al individuo en su interior a partir de relaciones de proximidad económica y cultural o de alejamiento en el desarrollo de las relaciones de poder, de manera que la cercanía física se vuelve también sinónimo de cercanía sociocultural (Bourdieu, 1997). De hecho, el reconocimiento planteado por Bourdieu (1997) se fundamenta a partir del capital cultural y económico poseído o según Hillmann y Riu (2001) a partir de la idea de estatus y por extensión de prestigio social.

En cambio, volviendo la mirada hacia el concepto de etnia, a su lado proponemos poner el concepto de origen nacional debido a que las entrevistas realizadas a las mujeres mexicanas cualificadas han evidenciado no sólo el alejamiento de todos aquellos símbolos étnicos que caracterizan los demás 
mexicanos generalmente procedentes del medio rural, sino también la formación de una identidad que se fundamenta en la nacionalidad para distinguirse de los connacionales que no pertenecen a su misma posición social. Así que, lo que se produce es un enfoque interseccional basado en el género, la posición social y el origen nacional/etnia.

\section{DATOS SOBRE LAS MUJERES MEXICANAS CUALIFICADAS QUE MIGRAN}

Un primer dato que necesitamos subrayar cuando nos referimos a las migraciones de las y los migrantes mexicanos, es el estereotipo según el cual la casi totalidad de los individuos involucrados en tales movimientos tendría escasos niveles de escolaridad. De este modo, la sobre representación de las y los migrantes mexicanos con un bajo perfil escolar invisibiliza la presencia en los flujos migratorios de los que, en cambio, son cualificados (Alarcón, 2001). Una invisibilidad que involucra también el mundo académico por la exigüidad de los estudios que han sido dedicados a la temática en cuestión (Albo y Ordaz Díaz, 2011).

$\mathrm{Si}$, al contrario, intentamos profundizar un poco más en estos flujos sabemos, a través de Albo y Ordaz Díaz(2011), que México tiene tasas de migración cualificada superiores a India, Irán, Brasil y Colombia, menores a las de Vietnam y Cuba, y similares a las de Filipinas. Del mismo modo, Calva Sánchez y Alarcón (2015) retomando datos de la American Community Survey señalan que entre 2009 y 2011 los migrantes mexicanos cualificados representaban el 31,6\% del total de los migrantes procedentes de México.

Volviendo ahora la mirada hacia las mujeres cualificadas, observamos que la escasez de datos se vuelve aún más sustancial debido a que «La falta de datos es parte de una falta general de reconocimiento y de interés a cerca de las mujeres migrantes cualificadas, por parte de académicos y responsables políticos ${ }^{2} »$. (Kofman y Raghuram, 2009, p. 3). Este desinterés puede entenderse como efecto de la lógica de género (De Oliveira y Ariza, 1999), según la cual las mujeres migran a partir de procesos de reagrupación familiar no finalizados a la inserción en el mercado laboral de los países de destino (Kofman y Raghuram, 2009). Sin embargo, cabe también subrayar que la invisibilidad de la cualificación de las mujeres mexicanas es parte de un proceso que se sustenta a partir de sobre representaciones. Una es la que se genera a través de la sobrerepresentación de las mujeres que se emplean en el servicio doméstico, considerando a priori esta inserción como efecto de bajos niveles escolares (Dumont, Martin y Spielvogel, 2007), mientras que la otra sobrerepresentación se realiza cuando la atención se concentra en los sectores laborales calificados donde los migrantes de género masculino son la mayoría, generando así la convicción de que los hombres presentan más altas tasas de cualificación (Kofman y Raghuram,2009).

Una condición desmentida por los datos debido a que en 2011 las mujeres representaban más de la mitad, a saber cerca del 52\%, del total de los migrantes mexicanos cualificados (Calva Sánchez, 2014).

\section{DINÁMICAS DE INCLUSIÓN Y EXCLUSIÓN}

Lo que hoy en día caracteriza la migración de mexicanos, hombres y mujeres, con o sin estudios, indígenas o no, es que tienen muy pocas probabilidades de insertarse en trabajos que no sean los de jardineros, camareros, empleados en el sector de la limpieza o trabajadoras del hogar, encarnando los estereotipos que guían su presencia en el mercado laboral (Donato, Gabaccia, Holdaway, Manalansan y Pessar 2006; Levine, 2006; Lozano Ascencio, Gandini y Ramírez-García, 2015). De hecho, si nos fijamos en la componente femenina de estos flujos migratorios sabemos que, según datos de 2014 , cerca del $26 \%$ de las migrantes mexicanas con un nivel universitario o más realizaba empleos no calificados y el 17,2\% desempeñaba ocupaciones de cuello blanco (Lozano Ascencio, Gandini y Ramírez García, 2015).

\footnotetext{
${ }^{2}$ Traducción propia.
} 
Ante esta situación, nos preguntamos porqué se produce esta condición paradójica y la respuesta nos viene de aquellos autores que insertan el fenómeno migratorio en el interior de mecanismos que producen nuevas formas de jerarquizaciones, de tal manera que lo que se produce de acuerdo con Sassen (1984), Boyd (1984) y Massey (2014) es la creación de un nuevo proletariado, de un underclass o de un new urban underclass respectivamente, mediados por la presencia de las y los migrantes que desarrollan el papel de mano de obra barata en un sistema de producción que se vuelve competitivo a partir del abaratamiento del coste del trabajo.

¿Y cómo se realiza este proceso? Pues, en este caso la repuesta tiene que tomar en cuenta más factores, el primero de los cuales es el género, debido a que la condición de las mujeres cualificadas no es igual a la de los hombres cualificados aunque el resultado sigue siendo para todos el del desclasamiento.

En este caso el enfoque interseccional nos ayuda a analizar el fenómeno desde múltiples niveles que involucran obviamente el género pero también los factores socioculturales, a saber la posición social y el origen nacional.

Empezando el análisis por la condición de género, cabe subrayar que el papel de las mujeres en el mercado laboral ha sido invisibilizado por el rol que, según la demarcación de los sexos, éstas tienen que desempeñar en el hogar como madres y esposas. De hecho, según la división de los espacios mientras los hombres se dedican a proveer los bienes necesarios para el sustento de la familia, ocupando el espacio público, las mujeres cuidan el hogar y procrean, ocupando el espacio privado (Benería,1981).

Ahora bien, si a estas reflexiones añadimos el análisis desde el punto de vista de la posición social, podemos observar que a partir del estereotipo bajo el cual todas las mujeres mexicanas presentan bajos niveles escolares, lo que se invisibiliza es la variedad sociocultural que en cambio caracteriza los flujos migratorios procedentes de México. Un fenómeno que se complejiza ulteriormente cuando en relación al origen nacional, diferentes autores han señalado mecanismos que han denominado la «desventaja de ser mexicano» (Angoa, 2009; Giorguli, Leite y Gaspar, 2007) y la "construcción de la ilegalidad mexicana» (De Genova, 2004) para resaltar, en el primer caso, aquellos estereotipos que participan en descalificar los flujos migratorios procedentes de México y, en el segundo caso, para mostrar la construcción de mecanismos que crean a priori una condición de desventaja. En este último caso a través de estrategias de cierre y apertura de las fronteras se consigue restringir o abrir el paso hacia el territorio norteamericano, creando un clima de miedo e inseguridad que influye en la inserción de las y los migrantes en el mercado laboral informal para evadir los controles de la policía de migración. El resultado es la constitución de mano de obra desprotegida ante los abusos y flexible ante las condiciones cambiantes del sistema productivo. En otras palabras, se asiste a la creación de mano de obra explotable.

\section{DISCUSIÓN Y CONCLUSIONES}

Concluimos esta discusión señalando que las estrategias adoptadas para volver migrantes cualificadas mano de obra a utilizar en empleos no calificados, invisibilizando al mismo tiempo su presencia en los circuitos formales, es parte de procesos de inclusión que operan mediante exclusión. ¿De qué manera? Primeramente a través de la deconstrucción del perfil sociolaboral de las mujeres mexicanas cualificadas a partir de la inivisibilización de las habilidades poseídas $y$, en segundo lugar, mediante la construcción de un nuevo perfil acorde a la posición social a ocupar en el lugar de asentamiento. Un proceso que se desliga del capital cultural poseído por las migrantes, a medida que la inclusión en el tejido sociolaboral de los lugares de llegada ya no se realiza según la formación profesional adquirida en el país de origen. De este modo, mujeres cualificadas se vuelven cuidadoras de ancianos y niños, camareras, cocineras, etc., ocupando todas aquellas posiciones que le son asignadas por el origen y el género. 
Así que, la exclusión que se realiza a partir de mecanismos descalificadores y de otros que ofuscan y precarizan la presencia de las migrantes mexicanas cualificadas en el lugar de asentamiento, produce su inclusión en el mercado laboral según un proceso que les asigna de manera ficticia un nuevo perfil sociolaboral. De este modo, las migrantes mexicanas que en el país de origen son profesionales ocupando estratos sociales medio-alto, en el país de asentamiento se vuelven parte de un nuevo proletariado conformado por los individuos procedentes de países que en la escala social se colocan en los eslabones más bajos.

\section{REFERENCIAS BIBLIOGRÁFICAS}

ALARCÓN, R. (2001). Immigrant niches in the US high-technology industry. The International Migration of the Highly Skilled. En W. A. Cornelius, T.J. Espenshade, and I. Salehyan (Eds.), The International Migration of the Highly Skilled: Demand, Supply, and demand consequences in sending and receiving countries (pp. 235-263). California: University of California-San Diego.

ALBO, A. y ORDAZ DÍAZ, J. L. (2011). La migración mexicana hacia los Estados Unidos: una breve radiografía. Documentos de trabajo. México: BBVA Research.

ANGOA, M. A. (2009). Mexicanas en Estados Unidos. El estado de la migración. En P. Leite y S. Giorguli (Eds.). Las políticas públicas ante los retos de la migración mexicana a Estados Unidos (pp. 171-210). México: CONAPO.

ANTHIAS, F. y YUVAL-DAVIS, N. (2010). Contextualizing feminism: gender, ethnic and class divisions. En M. Martiniello y J. Rath (Eds.), Selected Studies in International Migration and Immigrant Incorporation. Amsterdam: Amsterdam University Press.

BENERÍA, L. (1981), Reproducción, producción y división sexual del trabajo. Mientras Tanto, 6, 47-84.

BOURDIEU, P. (1997). Razones prácticas. Sobre la teoría de la acción. Barcelona: Editorial Anagrama.

BOYD, M. (1984). At a disadvantage: The occupational attainments of foreign born women in Canada. International Migration Review, 18 (4), 1091-1119.

CALVA SÁNCHEZ, L. E. y ALARCÓN, R. (2015). La integración laboral precaria de los migrantes mexicanos calificados en Estados Unidos al inicio del siglo XXI. Papeles de población, 21 (83), 9-39.

CALVA SÁNCHEZ, L. E. (2014). La migración calificada de mexicanos a Estados Unidos y su inserción al mercado laboral (Tesis de Doctorado). El Colegio de la Frontera Norte, México.

DE OLIVEIRA, O. y ARIZA, M. (1999). Trabajo, familia y condición femenina: una revisión de las principales perspectivas de análisis. Papeles de población, 5 (20), 89-127.

DONATO, K .M., GABACCIA, D., HOLDAWAY, J., MANALANSAN, M.y PESSAR, P. R. (2006). A glasshalffull? Genderinmigration studies. International migration review, 40 (1), 3-26. DUMONT, J. C., MARTIN, J. P. y SPIELVOGEL, G. (2007). Women on the move: the neglected gender dimension of the braindrain. IZA. Discussion Paper 2920.Recuperadode https://papers.ssrn.com/Sol3/papers.cfm?abstract_id=1001216.

GIORGULI, S., LEYTE, E. y GASPAR, S. (2007). La migración mexicana y el mercado de trabajo estadounidense, CONA$\mathrm{PO}$, Mexico.

HILLMANN, K. H.y RIU,G. M. (2001). Diccionario enciclopédico de sociología. Barcelona: Herder. KOFMAN, E. Y RAGHURAM, P. (2009). Skilled female labour migration. Policy Brief, 13,1-8.

LOZANO ASCENCIO, F., GANDINI, L. y RAMÍREZ-GARCÍA, T. (2015). Devaluación del trabajo de posgraduados en México y migración internacional: los profesionistas en ciencia y tecnología. Migración y desarrollo, 13, 61-89.

MASSEY, D. S. (2014). Manufacturing marginality among women and Latinos in neo-liberal America. Ethnic and racial studies, 37 (10), 1747-1752. 
SASSEN S. (1984). Notes on the incorporation of third world women into wage-labor through immigration and off-shoreproduction. International Migration Review, 18(4),1144-1167.

SEYMOUR-SMITH, C. (1986). Macmillan dictionary of anthropology. Londres: Macmillan. 